\title{
A field-oriented chain of dipolar particles in elongational flow
}

\author{
M.-Carmen Miguel ${ }^{1}$ and J.M. Rubí ${ }^{2}$ \\ ${ }^{1}$ Department of Physics \\ Massachusetts Institute of Technology, Cambridge, Massachusetts 02139 \\ ${ }^{2}$ Departament de Física Fonamental, Facultat de Física \\ Universitat de Barcelona \\ Diagonal 647, 08028 Barcelona, Spain
}

(November 14, 2017)

\begin{abstract}
We study the behavior of an isolated field-oriented chain of dipolar particles in elongational fluid flow. Our main goal is to emphasize the effect of dipolar interactions on the chain's contribution to the pressure tensor and to the viscosities of a dilute suspension of these linear aggregates. In our model, despite the overall rigid appearance of the chain at rest, the constituent beads may move slightly relative to one another, conferring a certain degree of flexibility to the chain. This flexibility is quantified in terms of a dimensionless parameter, $\lambda^{-1}$, comparing thermal and dipolar energies. We perform an expansion in $\lambda^{-1}$, and obtain the first correction to the rigid chain contribution to the Kramers' pressure tensor for different flow geometries. The interplay of the elongational flow field and the field-induced chain orientation gives rise to a rich variety of scenarios. We compute the elongational, shear, and rotational viscosities in some representative situations.
\end{abstract}

PACS numbers: 82.70.y, 83.80.Gv, 75.50.Mm, 05.40.+j

\section{INTRODUCTION}

Colloidal suspensions play an important role in many natural phenomena as well as in various industrial processes. The stability of these suspensions against aggregation of their constituent particles is of essential importance for their behaviond. As long as interparticle interactions can be neglected, one does not observe aggregation and the characterization of the system reduces to understanding how the physical properties of the fluid,e.g. its viscpsity - are modified due to the presence of the particles 1 . On the contrary, the phenomenology of the suspension may change dramatically if interactions between particles become relevant.

In the late 1930's, Winslow 6 first noted the curious behavior of a suspension of dielectric particles in oil when subjected to an electric field. He reported the formation of linear chains of particles aligned with the electric field, and how the effective viscosity of the suspension could change by orders of magnitude by simply modifying the applied field. An analogous field-induced behavior is exhibited by magnetorheological fluids, e.g., by a suspension of magnetizable superparamagnetic particles in a nonmagnetic fluid, or by a suspension of apnmagnetizable spheres in a ferrofluid (magnetic holes) 10 . Since then, several experimental and theoretical efforts, as well as computer simulations, have been devoted to study the different aspects of the complex hehavior of colloidal suspensions of dipolar particles 11 19.

The change in the rheological properties of dipolar suspensions upon the action of an external field, is in part due to the aggregation of the colloidal particles, which form clusters of macroscopic size. These are usually linear chains - quasi rigid rods - oriented along the direc- tion of the applied field, although for high enough concentrations of dipolar particles more complex structures may arise 2022. The overall spatial arrangement of the aggregates is very effective in hindering the fluid flow, conferring the suspension a solid-like texture.

Our purpose in this paper is to ascertain the role played by strong, but finite, dipolar interactions in the linear rheology of a suspension containing either an isolated field-induced aggregate or, equivalently, a dilute concentration of such clusters. After introducing the dimensionless parameter, $\lambda$, comparing dipolar and thermal energies, we obtain the first correction in powers of $\lambda^{-1}$ to the rigid $\operatorname{rod}\left(\lambda^{-1} \rightarrow 0\right)$ contribution to the Kramers' pressure tensor for different flow geometries. In particular, we analyze the influence that those interactions exert on the value of some of the transport coefficients of the system. We have organized the paper in the following way: Section II contains a description of the system and the conditions under study. The end-to-end vector of the chain is introduced and its mean value, as well as the mean square end-to-end distance, are obtained. In Section IIII we analyze the contribution of a chain, under the action of an elongational flow, to the pressure tensor of the suspension, and to some of the viscosities characterizing the system for different elongational flow fields of interest. The conclusions are summed up in the last section.

\section{DESCRIPTION OF THE SYSTEM}

The structure of a colloidal particle aggregate may be either fixed or deformable, depending upon the nature of the aggregation process and the type of interparticle 
bonds established. In Ref. 14, the issue of the growth of similar electric field-induced chains was experimentally addressed. There, the authors reported that, in the presence of an intense field, the colloidal dipoles organize themselves into linear chains. These clusters exhibit a certain degree of flexibility, limited of course by the strength of the dipolar interaction among the beads. Within one chain, they observed fluctuations in the separation between sphere surfaces $(5-10 \%$ of a diameter), as well as in the angle between the relative position vector of consecutive spheres and the applied field. Hence, we will assume that the beads forming the chain are in close contact, but still able to slightly move in relation to one another due to thermal fluctuations and to the action of an elongational flow field.

The dimensionless parameters describing the conditions of the system under study are essentially: $\lambda=$ $m^{2} /\left(d^{3} k_{B} T\right)$, comparing dipolar and thermal energies, and the Langevin parameter $\mu \equiv m H / k_{B} T$, where $m$ and $d$ are the dipolar moment and the diameter of each sphere, $H$ is the external (magnetic or electric) field strength, $k_{B}$ is the Boltzmann constant, and $T$ the absolute temperature 3 . In our analysis $\lambda$ is assumed to be large and $\mu \rightarrow \infty$, as in the experimental realizations in which the irreversible formation of these sort of quasi rigid linear aggregates is observed.

Despite the long-range contributions to the energy of a chain containing a fixed number of particles, $N$, the amplitude of the vibrations and oscillations of the spheres are almost entirely taken into account by imst considering interactions between nearest-neighbor 2222 . Long range effects are subleading corrections to the short range attractive contributions and can be ignored. As a matter of fact, the most important contributions correspond to relative distances among the particles of the order of one diameter, and relative orientations with an azimuthal angle, defined with respect to the external field direction, close to $\theta=0$.

Thus we will assume that the potential energy can be expressed as a sum of nearest neighbors terms

$$
\frac{V_{m a g}}{k_{B} T}=\sum_{i=1}^{N-1} \Phi_{i, i+1} .
$$

Here,

$$
\Phi_{i, j}=\frac{\boldsymbol{m}_{i} \cdot \boldsymbol{m}_{j}-3\left(\boldsymbol{m}_{i} \cdot \hat{\boldsymbol{r}}_{i j}\right)\left(\boldsymbol{m}_{j} \cdot \hat{\boldsymbol{r}}_{i j}\right)}{r_{i j}^{3}}
$$

is the interaction energy of the dipoles $i$ and $j$, with $\boldsymbol{r}_{i j}=r_{i j} \hat{\boldsymbol{r}}_{i j}$ the vector giving their relative position. Its modulus $r_{i j}$ is the distance between the sphere centers. Since the most important contributions come from distances $r_{i j} \sim d$ and relative orientations $\theta_{i j} \sim 0$, each term in the sum of Eq. (1) can be approximately written as follows20:

$$
\Phi_{i, i+1} \sim-\lambda\left(2-3 \theta_{i}^{2}-6 \xi_{i}\right) .
$$

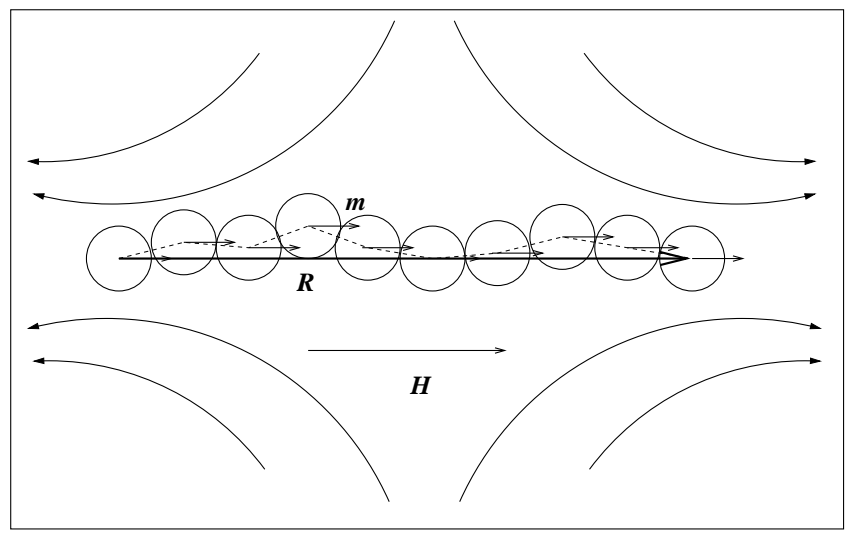

FIG. 1. Chain of magnetic particles in an elongational flow. All the magnetic moments $\boldsymbol{m}$ point towards the direction of the external field applied. The vector $\boldsymbol{R}$ is the end-to-end vector giving the relative position of the centers of the spheres on the extremes.

In the last equation, we have introduced the relative position vector between two consecutive spheres in the chain $\boldsymbol{q}_{i}=\boldsymbol{R}_{i+1}-\boldsymbol{R}_{i}$ through its components in spherical coordinates $\boldsymbol{q}_{i} \equiv\left(q_{i}, \theta_{i}, \varphi_{i}\right)$. The position vector $\boldsymbol{R}_{i}$ determines the center of the $i$-th sphere. Moreover, we have performed an expansion around the minimum of the energy in the variables $\theta_{i}$ and $\xi_{i}$, which, in turn, is defined by $q_{i}=d\left(1+\xi_{i}\right)$, and such that $0 \leq \xi_{i} \ll 1$.

Multipole and multibody contributions are not considered because they are expected to be very small. Likewise, we disregard London-Van der Waals forces, and any possible steric interactions due to the surfactant monolayer with which the colloidal particles may be usually coated.

\section{Mean size of the chain}

To characterize the mean size of a chain, containing $N$ dipoles, under the action of an elongational flow and thermal fluctuations, let us consider the end-to-end vector $\boldsymbol{R}$ giving the relative position of the centers of the spheres located on the tips of the chain (see Fig. 1)

$$
\boldsymbol{R}=\boldsymbol{R}_{N}-\boldsymbol{R}_{1}=\sum_{k=1}^{N-1} \boldsymbol{q}_{k} .
$$

In order to compute the average of this vector, or equivalently of the vector $\boldsymbol{q}_{k}$, we need the probability distribution $\psi\left(\left\{\boldsymbol{R}_{i}\right\}\right)$ of the spheres' position. This probability is the solution of the diffusion equation describing the com servation of system points in the configurational space 24,

$$
\begin{aligned}
\frac{\partial \psi}{\partial t} & =\sum_{i}\left\{\frac{\partial}{\partial \boldsymbol{R}_{i}} \cdot \sum_{j} \boldsymbol{\mu}_{i j}^{T T} \cdot\left(k_{B} T \frac{\partial \psi}{\partial \boldsymbol{R}_{j}}+\frac{\partial V_{m a g}}{\partial \boldsymbol{R}_{j}} \psi\right)\right. \\
& \left.-\frac{\partial}{\partial \boldsymbol{R}_{i}} \cdot\left(\boldsymbol{\beta} \cdot \boldsymbol{R}_{i}\right) \psi\right\},
\end{aligned}
$$


where $\boldsymbol{\mu}_{i j}^{T T}$ is the relative translational mobility tensor, $V_{m a g}$ is the dipole-dipole magnetic potential, and $\boldsymbol{\beta}$ is the elongational rate tensor $\left(\boldsymbol{\beta}=\boldsymbol{\beta}^{T}\right)$ corresponding to the stationary homogeneous external flow, $\boldsymbol{v}_{o}=\boldsymbol{\beta} \cdot \boldsymbol{r}$. As an approximation, we assume that the spheres move through the solvent without disturbing the velocity field (free draining), so that

$$
\boldsymbol{\mu}_{i j}^{T T}=\frac{1}{6 \pi \eta a} \boldsymbol{I} \delta_{i j}
$$

where, for the sake of simplicity, we consider that all the spheres in the chain have the same radius $a=d / 2$, and $\boldsymbol{I}$ is the unit tensor.

The next step is to write the diffusion equation in terms of the relative position vectors, $\boldsymbol{q}_{i}$. To this purpose we introduce the transformation 25

$$
\boldsymbol{q}_{i}=\sum_{k} B_{i k} \boldsymbol{R}_{k}, \quad \text { with } \quad B_{i k}=\delta_{i+1, k}-\delta_{i, k},
$$

from which one infers the relation $\partial / \partial \boldsymbol{R}_{i}=$ $\sum_{k} B_{k i} \partial / \partial \boldsymbol{q}_{k} ;$ and the matrix

$$
A_{i j}=\sum_{k} B_{i k} B_{j k}= \begin{cases}2 & \text { if } i=j \\ -1 & \text { if } i=j \pm 1 \\ 0 & \text { otherwise }\end{cases}
$$

Taking into account Eqs. (6)-(8), the diffusion equation can be alternatively written as

$$
\begin{aligned}
& \frac{\partial \psi}{\partial t}=\frac{k_{B} T}{6 \pi \eta a} \sum_{j} \frac{\partial}{\partial \boldsymbol{q}_{j}} \cdot \sum_{k} A_{j k}\left(\frac{\partial \psi}{\partial \boldsymbol{q}_{k}}+\frac{\partial V_{m a g} / k_{B} T}{\partial \boldsymbol{q}_{k}} \psi\right) \\
& \quad-\sum_{j} \frac{\partial}{\partial \boldsymbol{q}_{j}} \cdot\left(\boldsymbol{\beta} \cdot \boldsymbol{q}_{j}\right) \psi .
\end{aligned}
$$

The stationary solution of this equation with a homogeneous potential flow field is

$$
\begin{array}{r}
\psi_{s t}\left(\boldsymbol{q}_{1}, \ldots, \boldsymbol{q}_{N-1}\right) \sim \exp \left\{\frac{-V_{m a g}}{k_{B} T}\right. \\
\left.+\frac{3 \pi \eta a}{k_{B} T}\left(\boldsymbol{\beta}: \sum_{i} \sum_{j} C_{i j} \boldsymbol{q}_{i} \boldsymbol{q}_{j}\right)\right\},
\end{array}
$$

where the symbol : indicates a double contraction of indexes, and $C_{i j}$ is the Kramers matrix defined in Ref. 25. Up to linear order in the elongational rate $\boldsymbol{\beta}$ linear dynamics - , we expand the exponential factor in Eq. (10). The stationary average of $\boldsymbol{q}_{k}$ is then given by

$$
\begin{aligned}
\left\langle\boldsymbol{q}_{k}\right\rangle & =\int\left(\prod_{l=1}^{N-1} d \boldsymbol{q}_{l}\right) \boldsymbol{q}_{k} \psi_{s t} \simeq d \hat{\boldsymbol{e}}_{z} \\
& +\frac{d^{3}}{6 D} \lambda^{-1}\left(\boldsymbol{\beta} \cdot \hat{\boldsymbol{e}}_{z}-\beta_{z z} \hat{\boldsymbol{e}}_{z}\right) \sum_{i} C_{i k}
\end{aligned}
$$

where we have introduced the translational diffusion coefficient for a single particle $D=k_{B} T /(6 \pi \eta a)$. This result is obtained after decomposing the integral into different parts, and taking into account the following relations 25 :

$$
\sum_{i=1}^{N-1} \sum_{j=1}^{N-1} C_{i j}=\frac{N\left(N^{2}-1\right)}{12}, \quad \sum_{i=1}^{N-1} C_{i i}=\frac{N^{2}-1}{6} .
$$

In the Appendix we indicate in more detail some of the calculations involved in the computation of a similar average which appears in the following section.

Therefore, up to first order in $\lambda^{-1}$ and in the elongational flow rate, the average end-to-end vector $\boldsymbol{R}$ defined in Eq. (4) turns out to be

$$
\langle\boldsymbol{R}\rangle \simeq(N-1) d \hat{\boldsymbol{e}}_{z}+N\left(N^{2}-1\right) \frac{d^{3}}{72 D} \lambda^{-1}\left(\boldsymbol{\beta} \cdot \hat{\boldsymbol{e}}_{z}-\beta_{z z} \hat{\boldsymbol{e}}_{z}\right)
$$

where, once again, use has been made of the relations (12). At equilibrium $(\boldsymbol{\beta}=0)$, this average obviously reduces to $\langle\boldsymbol{R}\rangle_{e q}=(N-1) d \hat{\boldsymbol{e}}_{z}$ - the length of a rigid chain.

Following the same procedure we can compute the mean square end-to-end distance

$$
\left\langle R^{2}\right\rangle=\langle\boldsymbol{R} \cdot \boldsymbol{R}\rangle=\sum_{k=1}^{N-1} \sum_{m=1}^{N-1}\left\langle\boldsymbol{q}_{k} \cdot \boldsymbol{q}_{m}\right\rangle
$$

Up to first order in $\beta$, and in the small parameter $\lambda^{-1}$ of our expansion, we do not find any contribution from the elongational rate. Nevertheless, the equilibrium value is given by

$$
\left\langle R^{2}\right\rangle_{e q} \simeq(N-1)^{2} d^{2}+\frac{(N-1)}{3} \lambda^{-1} d^{2} .
$$

Thermal fluctuations effectively stretch a quasi rigid field-oriented chain-using the root mean square endto-end distance as a measure of its length - by a global amount $[(N-1) /(3 \lambda)]^{1 / 2} d$. On the other hand, thermal effects would be obviously negligible for a rigid chain $(\lambda \gg N)^{26}$. The stretching factor per bond is

$$
\frac{\left[\left\langle R^{2}\right\rangle_{e q}-\langle\boldsymbol{R}\rangle_{e q}^{2}\right]^{1 / 2}}{N-1}=[3 \lambda(N-1)]^{-1 / 2} d .
$$

Thus, for large but finite values of $\lambda N$, i.e. $(\lambda N)^{-1} \neq 0$, the mean separation between consecutive spheres increases by a factor proportional to $(\lambda N)^{-1 / 2}$. This result (16) is in qualitative and quantitative agreement with the observations reported in Ref. 14 for similar electric fieldinduced chains. In these experiments, $\lambda \sim(20-30)$ and the mean number of particles in a chain at the late stages of the aggregation process is typically $N \sim(5-15)$ which, according to our result (16), yield a mean near-neighbors separation of about $(3-6) \%$ of $d$. This value is comparable to their observations, in which the sphere surfaces appear to be separated by $(5-10) \%$ of a sphere diameter due to thermal fluctuations. 


\section{CONTRIBUTION OF THE CHAIN TO THE PRESSURE TENSOR}

Besides the contribution of the solvent to the total pressure tensor of the suspension, there is another contribution coming from the direct interaction of the particles constituting the chains. The latter can be obtained from theological equation of state proposed by Kramers25.27

$$
\boldsymbol{\Pi}^{p}=-\frac{1}{V} \sum_{k=1}^{N-1}\left\langle\boldsymbol{q}_{k} \frac{\partial V_{m a g}}{\partial \boldsymbol{q}_{k}}\right\rangle+\frac{N-1}{V} k_{B} T \boldsymbol{I},
$$

where $V$ is the volume of the system.

Once we know the stationary solution of the diffusion equation (10), the average appearing on the right hand side of equation (17) can be expressed as an integral of the form:

$$
\left\langle\boldsymbol{q}_{k} \frac{\partial V_{m a g}}{\partial \boldsymbol{q}_{k}}\right\rangle=\int \prod_{l=1}^{N-1} d \boldsymbol{q}_{l}\left(\boldsymbol{q}_{k} \frac{\partial V_{m a g}}{\partial \boldsymbol{q}_{k}}\right) \psi_{s t}\left(\boldsymbol{q}_{1}, \ldots, \boldsymbol{q}_{N-1}\right) .
$$

As we are interested in finding the Newtonian viscosity tensor, we again expand the exponential factor in Eq. (10) up to first order in $\boldsymbol{\beta}$. To evaluate the remaining integral, it is convenient to decompose it into different parts as indicated in the Appendix; thereby, in the Newtonian domain, the average in Eq. (18) reads

$$
\begin{aligned}
& \left\langle\boldsymbol{q}_{k} \frac{\partial V_{m a g}}{\partial \boldsymbol{q}_{k}}\right\rangle \simeq k_{B} T\left\{\boldsymbol{I}+\frac{d^{2}}{D} \sum_{i} C_{i k} \boldsymbol{\beta} \cdot \hat{\boldsymbol{e}}_{z} \hat{\boldsymbol{e}}_{z}\right. \\
& \left.+\frac{d^{2}}{6 D} \lambda^{-1} C_{k k} \boldsymbol{\beta} \cdot\left(\boldsymbol{I}-\hat{\boldsymbol{e}}_{z} \hat{\boldsymbol{e}}_{z}\right)\right\} .
\end{aligned}
$$

Finally, after using Eqs. (12), we obtain the contribution of one chain to the pressure tensor (17)

$\boldsymbol{\Pi}^{p} \simeq-\frac{\pi \eta_{o} d^{3}}{4 V} N\left(N^{2}-1\right)\left[\hat{\boldsymbol{e}}_{z} \hat{\boldsymbol{e}}_{z}+(3 \lambda N)^{-1}\left(\boldsymbol{I}-\hat{\boldsymbol{e}}_{z} \hat{\boldsymbol{e}}_{z}\right)\right] \cdot \boldsymbol{\beta}$.

The term proportional to $(\lambda N)^{-1}$ constitutes the first correction to the rigid chain limit $\left(\lambda^{-1} \rightarrow 0\right)$. Depending upon the structure of the flow rate, this term represents either an increase or a decrease in the value of the different components of the pressure tensor as a function of $\lambda$, i.e. when the chain becomes more flexible. Note that, as we are not taking into account hydrodynamic interactions between the particles (free draining approximation), the rigid chain-limiting pressure tensor grows simply as the third power of the chain length. Hydrodynamic interactions are responsible for the logarithmic term that should also appear when dealing with a long straight line of spheres27, but their effects are less important for shorter chains as the ones considered here.
Moreover, from this expression we can obtain the contribution of the chain to the viscosity tensor, $\boldsymbol{\eta}_{p}$, which we can identify by comparing with the relationship

$$
\boldsymbol{\Pi}^{p}=-\boldsymbol{\eta}_{p}: \boldsymbol{\beta} .
$$

This tensor is thus

$\boldsymbol{\eta}_{p} \simeq \frac{\pi \eta_{o} d^{3}}{4 V} N\left(N^{2}-1\right)\left\{\hat{\boldsymbol{e}}_{z} \boldsymbol{I} \hat{\boldsymbol{e}}_{z}+(3 \lambda N)^{-1}\left(\boldsymbol{S}-\hat{\boldsymbol{e}}_{z} \boldsymbol{I} \hat{\boldsymbol{e}}_{z}\right)\right\}$

where $\boldsymbol{S}$ is the fourth-rank tensor $S_{i j k l}=\left(\delta_{i k} \delta_{j l}+\right.$ $\left.\delta_{i l} \delta_{j k}\right) / 2$, symmetric in any of these index transformations $(i \leftrightarrow j, k \leftrightarrow l,(i j) \leftrightarrow(k l))$.

For the conditions under consideration, the chains are always oriented in the direction of the field, but smoothly vibrate and oscillate around this orientation. In this case, the symmetries of the fluid flow essentially determine the characteristics of both the pressure and the viscosity tensors. For instance, as the chains are oriented along the z-axis - parallel to the external field - if the flow field rate $\boldsymbol{\beta}$ is diagonal, so will be the pressure tensor. On the other hand, for a non-diagonal flow rate, we have, in general, both symmetric and antisymmetric contributions. Let us illustrate the structure of the pressure tensor by considering some representative cases of interest:

i) Flow through a pore. In this case, if the system has rotational symmetry around the $z$-axis, the velocity field is given by

$$
v_{x}=-\frac{1}{2} \beta x \quad v_{y}=-\frac{1}{2} \beta y \quad v_{z}=\beta z,
$$

where $\beta$ is the elongational rate. The pressure tensor has then the following form

$\boldsymbol{\Pi}^{p} \simeq-\frac{\pi \eta_{o} d^{3}}{4 V} N\left(N^{2}-1\right)\left(\begin{array}{ccc}-(6 \lambda N)^{-1} & 0 & 0 \\ 0 & -(6 \lambda N)^{-1} & 0 \\ 0 & 0 & 1\end{array}\right) \beta$

Under these conditions the chain is subjected to tensile and compressive forces giving rise to the so-called elongational viscosities defined from the differences between two of the diagonal components of the pressure tensor, i.e. normal pressure differences. As an example,

$$
-\frac{\Pi_{z z}^{p}-\Pi_{x x}^{p}}{\beta} \simeq \frac{\pi \eta_{o} d^{3}}{4 V} N\left(N^{2}-1\right)\left[1+(6 \lambda N)^{-1}\right] .
$$

This quantity essentially grows with the third power of the rigid chain length $(N d)^{3}$, and for high but finite values of $\lambda$. When $\lambda^{-1} \neq 0$, the chain becomes slightly more flexible, its effective length increases giving rise to a small increment in the viscosity of the system.

Alternatively, a fluid flow with rotational symmetry around the $x$ (or $y$ ) axis leads to the following structure of the pressure tensor: 
$\Pi^{p} \simeq-\frac{\pi \eta_{o} d^{3}}{8 V} N\left(N^{2}-1\right)\left(\begin{array}{ccc}2(3 \lambda N)^{-1} & 0 & 0 \\ 0 & -(3 \lambda N)^{-1} & 0 \\ 0 & 0 & -1\end{array}\right)$

Combinations of any two diagonal components of this tensor amount to different elongational viscosity coefficients. Moreover, in any of the above mentioned geometries, we have another viscosity coefficient coming from the trace of the pressure tensor.

ii) Planar elongational flow. This flow can be generated by four rotating cylinders. If we locate the cylinders such that the elongational tensor is again a diagonal matrix, in view of Eq. (20), the pressure tensor is also diagonal and, of course, symmetric. For example, if

$$
v_{x}=-\beta x \quad v_{z}=\beta z,
$$

we find a similar phenomenology as the one described above. On the other hand, in a reference frame rotated $45^{\circ}$ with respect to the previous one, the elongational tensor is

$$
\boldsymbol{\beta}=\beta\left(\begin{array}{ccc}
-1 & 0 & 1 \\
0 & 0 & 0 \\
1 & 0 & 1
\end{array}\right)
$$

resulting in

$$
\Pi^{p} \simeq-\frac{\pi \eta_{o} d^{3}}{4 V} N\left(N^{2}-1\right)\left(\begin{array}{ccc}
-(3 \lambda N)^{-1} & 0 & 1 \\
0 & 0 & 0 \\
(3 \lambda N)^{-1} & 0 & 1
\end{array}\right) \beta,
$$

which has both symmetric and antisymmetric contributions. Related to these parts, we find not only elongational viscosities but also a shear viscosity, $\eta$, and a rotational viscosity, $\eta_{r}$. These last quantities can be identified from the non-diagonal components of the symmetric and antisymmetric parts, $\Pi_{x z}^{p(s)}$ and $\Pi_{x z}^{p(a)}$, respectively. They turn out to be

$$
\begin{aligned}
\eta & \simeq \frac{\pi \eta_{o} d^{3}}{16 V} N\left(N^{2}-1\right)\left[1+(3 \lambda N)^{-1}\right] \\
\eta_{r} & \simeq \frac{\pi \eta_{o} d^{3}}{16 V} N\left(N^{2}-1\right)\left[1-(3 \lambda N)^{-1}\right] .
\end{aligned}
$$

Thus, for $\lambda^{-1} \rightarrow 0$, both shear and rotational viscosities behave like those of a dilute suspension of cylinders and grow with the third power of the rigid chain length, $(N d)^{3}$. For high but finite values of $\lambda$, however, the shear viscosity increases, whereas the rotational viscosity decreases. The reason why the latter occurs is that in this particular geometry, the elongational flow not only stretches the chain but also tends to reorient it. This rotation is opposed by the presence of the external field; in the case of a rigid chain $\left(\lambda^{-1} \rightarrow 0\right)$, the magnetic field impedes more effectively the reorientation and, as a consequence, the rotational viscosity attains its maximum value. Nonetheless, the rotational viscosity is smaller for $\beta$. a finite value of the parameter $\lambda^{-1}$, or, in other words, for a slightly more flexible chain. In Fig. 2 we plot the relative variation of the shear viscosity as a function of the number of particles in the chain $N$, for three given values of $\lambda^{-1}=0.01,0.05,0.1$ within the experimental range. If we define the shear viscosity in the $\lambda^{-1} \rightarrow 0$ limit as $\eta^{0}$, the relative correction $\left(\eta-\eta^{0}\right) / \eta^{0}=(3 \lambda N)^{-1}$ grows linearly with $\lambda^{-1}$, and becomes less important for longer chains. The relative decrease of the rotational viscosity $\left|\eta_{r}-\eta_{r}^{0}\right| / \eta_{r}^{0}$ shows the same behavior depicted in Fig. 2.

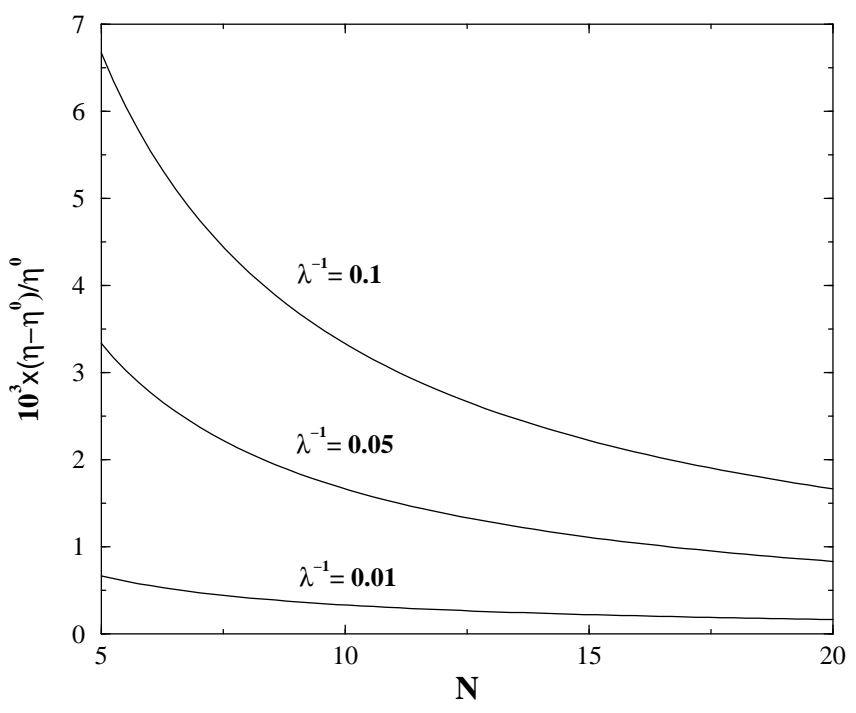

FIG. 2. Relative correction to the shear viscosity of a chain as a function of the number of dipolar particles $N$, for fixed values of $\lambda^{-1}=0.01,0.05,0.1$.

\section{CONCLUSIONS}

In summary, we have computed the contribution of a linear chain of dipolar particles to the Kramers' pressure tensor and the viscosities of a dilute suspension of such clusters. The field-induced chain is oriented along a preferred direction - parallel to the external field - but, at the same time, is exposed to both thermal effects and the influence of an external elongational fluid flow.

As was pointed out in previous experimental studies, these linear clusters exhibit a certain degree of flexibility despite its rod-like appearance. At equilibrium, thermal fluctuations are responsible for average separations between the spheres surfaces of the order of $(5-10) \%$ of a diameter, as well as for slight angular deviations, which amount to an effectively larger mean square end-to-end length of these field-oriented chains. The flexibility of a chain is obviously limited by the strength of the dipolar interactions among its constituent colloidal particles. The dimensionless parameter $\lambda$, comparing dipolar and 
thermal energies, thus becomes an essential ingredient in our analysis.

The rigid chain-like rheologic behavior, which corresponds to the limit $\lambda^{-1} \rightarrow 0$, is modified considerably as the chains become more flexible, i.e., for large but finite values of $\lambda$. Firstly, we have characterized the mean size of the chain obtaining the average end-to-end vector and the mean square end-to-end distance as a function of the parameter $\lambda^{-1}$. Secondly, we have computed the contribution of the chain to the pressure tensor of the system from the rheological equation of state proposed by Kramers. The structure of the pressure tensor has been explicitly illustrated for different elongational flows. Furthermore, by comparing our result to the standard linear law relating the pressure tensor and the elongational flow rate, we have calculated the viscosity tensor of the system.

Some of the most common viscosity coefficients characterizing a colloidal suspension of this kind have also been obtained. We have discussed the role played by the parameter $\lambda$ in the behavior of these quantities for two practical situations of interest: a flow through a pore and a planar elongational flow. In the first one, the symmetry of the contribution of the chains to the pressure tensor of the suspension only gives rise to elongational viscosities, defined from the differences between any pair of diagonal components of the pressure tensor. As the flow field effectively stretches the chain by a global amount proportional to $\lambda^{-1}$, the elongational viscosity also increases by a similar factor beyond its rigid limit value. In the second situation described, the chain's contribution to the pressure tensor is no longer symmetric; in this case, we have computed the so-called shear and rotational viscosities. The former increases with $\lambda^{-1}$ as well, whereas the latter decreases, i.e., the reorientation of the chains is slightly favored for large but finite values of this parameter, yielding a rotational viscosity smaller than in the case of a rigid rod.

Our results, valid up to the orders specified in the analysis, should provide a good description of the most frequent experimental conditions and, consequently, could be directly contrasted with experiments.

\section{ACKNOWLEDGMENTS}

We are grateful to Dr. R. Pastor-Satorras for his critical reading of the manuscript. M. C. M. was supported by a grant from the Direcció General de Recerca (Generalitat de Catalunya) and by the NSF Grant No. DMR93-03667. We also acknowledge financial support by the DGICYT of the Spanish Government (Grant No. PB950881).

\section{APPENDIX:}

In this appendix, we indicate in some detail some of the steps involved in the evaluation of Eq. (18). First, we split up the integral into different parts, a)-e), which are
a) $\int\left(\prod_{l=1}^{N-1} d \boldsymbol{q}_{l} \exp \left(-\Phi_{l, l+1}\right)\right)\left(\boldsymbol{q}_{k} \frac{\partial V_{\text {mag }}}{\partial \boldsymbol{q}_{k}}\right)$
b) $\frac{\boldsymbol{\beta}}{2 D}: \sum_{i(i \neq k)} \sum_{j(j \neq i, k)} C_{i j} \int\left(\prod_{l=1}^{N-1} d \boldsymbol{q}_{l} \exp \left(-\Phi_{l, l+1}\right)\right) \boldsymbol{q}_{i} \boldsymbol{q}_{j}\left(\boldsymbol{q}_{k} \frac{\partial V_{m a g}}{\partial \boldsymbol{q}_{k}}\right)$
c) $\frac{\boldsymbol{\beta}}{2 D}: \sum_{i(i \neq k)} C_{i i} \int\left(\prod_{l=1}^{N-1} d \boldsymbol{q}_{l} \exp \left(-\Phi_{l, l+1}\right)\right) \boldsymbol{q}_{i} \boldsymbol{q}_{i}\left(\boldsymbol{q}_{k} \frac{\partial V_{\text {mag }}}{\partial \boldsymbol{q}_{k}}\right)$
d) $\frac{\boldsymbol{\beta}}{2 D}: \sum_{i(i \neq k)} C_{i k} \int\left(\prod_{l=1}^{N-1} d \boldsymbol{q}_{l} \exp \left(-\Phi_{l, l+1}\right)\right) \boldsymbol{q}_{i} \boldsymbol{q}_{k}\left(\boldsymbol{q}_{k} \frac{\partial V_{\text {mag }}}{\partial \boldsymbol{q}_{k}}\right)$
e) $\frac{\boldsymbol{\beta}}{2 D}: C_{k k} \int\left(\prod_{l=1}^{N-1} d \boldsymbol{q}_{l} \exp \left(-\Phi_{l, l+1}\right)\right) \boldsymbol{q}_{k} \boldsymbol{q}_{k}\left(\boldsymbol{q}_{k} \frac{\partial V_{\text {mag }}}{\partial \boldsymbol{q}_{k}}\right)$.

Let us explicitly compute some of these contributions. For instance,

$$
\text { a) } \begin{aligned}
& \int\left(\prod_{l=1}^{N-1} d \boldsymbol{q}_{l} \exp \left(-\Phi_{l, l+1}\right)\right)\left(\boldsymbol{q}_{k} \frac{\partial V_{m a g}}{\partial \boldsymbol{q}_{k}}\right) \\
= & {\left[\int d \boldsymbol{q}_{k} \exp \left(-\Phi_{k, k+1}\right)\right]^{N-2} \int d \boldsymbol{q}_{k} \boldsymbol{q}_{k} \frac{\partial \Phi_{k, k+1}}{\partial \boldsymbol{q}_{k}} \exp \left(-\Phi_{k, k+1}\right) }
\end{aligned}
$$




$$
\begin{aligned}
\simeq & {\left[2 \pi \int_{0}^{\infty} q_{k}^{2} d q_{k} \int_{0}^{\pi} \sin \theta_{k} d \theta_{k} e^{\lambda\left(2-3 \theta_{k}^{2}-6 \xi_{k}\right)}\right]^{N-1} k_{B} T \boldsymbol{I} } \\
\simeq & {\left[2 \pi d^{3} e^{2 \lambda} \int_{0}^{\infty}\left(1+2 \xi_{k}\right) d \xi_{k} e^{-6 \lambda \xi_{k}} \int_{0}^{\infty}\left(\theta_{k}-\frac{\theta_{k}^{3}}{3 !}\right) d \theta_{k} e^{-3 \lambda \theta_{k}^{2}}\right]^{N-1} k_{B} T \boldsymbol{I} } \\
\simeq & {\left[\frac{\pi d^{3} e^{2 \lambda}}{18 \lambda^{2}}\left(1+\frac{5}{18 \lambda}\right)\right]^{N-1} k_{B} T \boldsymbol{I}, } \\
\text { b) } & \frac{\boldsymbol{\beta}}{2 D}: \sum_{i(i \neq k)} \sum_{j(j \neq i, k)} C_{i j} \int\left(\prod_{l=1}^{N-1} d \boldsymbol{q}_{l} \exp \left(-\Phi_{l, l+1}\right)\right) \boldsymbol{q}_{i} \boldsymbol{q}_{j}\left(\boldsymbol{q}_{k} \frac{\partial V_{m a g}}{\partial \boldsymbol{q}_{k}}\right) \\
\simeq & {\left[\frac{\pi d^{3} e^{2 \lambda}}{18 \lambda^{2}}\left(1+\frac{5}{18 \lambda}\right)\right]^{N-3} \frac{\boldsymbol{\beta}}{2 D}: \sum_{i(i \neq k)} \sum_{j(j \neq i, k)} C_{i j} \times } \\
& {\left[\int d \boldsymbol{q}_{i} \exp \left(-\Phi_{i, i+1}\right) \boldsymbol{q}_{i}\right]^{2} \int d \boldsymbol{q}_{k} \boldsymbol{q}_{k} \frac{\partial \Phi_{k, k+1}}{\partial \boldsymbol{q}_{k}} \exp \left(-\Phi_{k, k+1}\right) } \\
\simeq & {\left[\frac{\pi d^{3} e^{2 \lambda}}{18 \lambda^{2}}\left(1+\frac{5}{18 \lambda}\right)\right]^{N-1} \frac{k_{B} T d^{2} \beta_{z z}}{2 D} \sum_{i(i \neq k)} \sum_{j(j \neq i, k)} C_{i j} \boldsymbol{I} . }
\end{aligned}
$$

The other contributions are obtained in a similar fashion. Adding up the different parts and considering the relations (12), we can rewrite the average in Eq. (18) as

$$
\begin{aligned}
& \left\langle\boldsymbol{q}_{k} \frac{\partial V_{m a g}}{\partial \boldsymbol{q}_{k}}\right\rangle=C\left[\frac{\pi d^{3} e^{2 \lambda}}{18 \lambda^{2}}\left(1+\frac{5}{18 \lambda}\right)\right]^{N-1} k_{B} T\left\{\left[1+\frac{d^{2} \beta_{z z}}{24 D} N\left(N^{2}-1\right)\right] \boldsymbol{I}\right. \\
& \left.\quad+\frac{d^{2}}{D} \sum_{i} C_{i k} \boldsymbol{\beta} \cdot \hat{\boldsymbol{e}}_{z} \hat{\boldsymbol{e}}_{z}+\frac{d^{2}}{12 D} \lambda^{-1}\left[\boldsymbol{\beta}:\left(\boldsymbol{I}-\hat{\boldsymbol{e}}_{z} \hat{\boldsymbol{e}}_{z}\right) \frac{N^{2}-1}{6} \boldsymbol{I}+2 C_{k k} \boldsymbol{\beta} \cdot\left(\boldsymbol{I}-\hat{\boldsymbol{e}}_{z} \hat{\boldsymbol{e}}_{z}\right)\right]\right\} .
\end{aligned}
$$

Finally, after the appropriate normalization of the probability density, and up to first order in $\boldsymbol{\beta}$, we obtain

$$
\begin{aligned}
\left\langle\boldsymbol{q}_{k} \frac{\partial V_{\text {mag }}}{\partial \boldsymbol{q}_{k}}\right\rangle & =k_{B} T\left\{\left[1+\frac{d^{2} \beta_{z z}}{24 D} N\left(N^{2}-1\right)\right] \boldsymbol{I}+\frac{d^{2}}{D} \sum_{i} C_{i k} \boldsymbol{\beta} \cdot \hat{\boldsymbol{e}}_{z} \hat{\boldsymbol{e}}_{z}\right. \\
& \left.+\frac{d^{2}}{12 D} \lambda^{-1}\left[\boldsymbol{\beta}:\left(\boldsymbol{I}-\hat{\boldsymbol{e}}_{z} \hat{\boldsymbol{e}}_{z}\right) \frac{N^{2}-1}{6} \boldsymbol{I}+2 C_{k k} \boldsymbol{\beta} \cdot\left(\boldsymbol{I}-\hat{\boldsymbol{e}}_{z} \hat{\boldsymbol{e}}_{z}\right)\right]\right\} \\
& \times\left\{1-\frac{d^{2} \beta_{z z}}{24 D} N\left(N^{2}-1\right)-\frac{d^{2}}{72 D} \lambda^{-1}\left(N^{2}-1\right) \boldsymbol{\beta}:\left(\boldsymbol{I}-\hat{\boldsymbol{e}}_{z} \hat{\boldsymbol{e}}_{z}\right)\right\},
\end{aligned}
$$

which can be simplified to the final form (19).

${ }^{1}$ A.P. Gast and C.F. Zukoski, Adv. Colloid Interface Sci. 30, 153 (1989).

2 T.G.M. van de Ven, Colloidal Hydrodynamics, (Academic Press, London, 1989).

${ }^{3}$ M.I. Shliomis, Sov. Phys. JETP 34, 1291 (1972).

${ }^{4}$ M.C. Miguel and M. Rubí, Phys. Rev. E 51, 2190 (1995).

${ }^{5}$ J.-C. Bacri, R. Perzynski, M.I. Shliomis and G.I. Burde, Phys. Rev. Lett. 75, 2128 (1995).

${ }^{6}$ W.M. Winslow, J. Appl. Phys. 20, 1137 (1949).

${ }^{7}$ G. Bossis, C. Mathis, Z. Mimouni, and C. Paparoditis, Eu-

rophys. Lett. 11, 133 (1990).

${ }^{8}$ A.T. Skjeltorp, Phys. Rev. Lett. 51, 2306 (1983); J. Magn. Magn. Mat. 65, 195 (1987); J. Appl. Phys. 57 (1), 3285 (1985).

${ }^{9}$ P. Davies, J. Popplewell, G. Martin, A. Bradbury and R.W. Chantrell, J. Phys. D, Appl. Phys. 19, 469 (1986).

${ }^{10}$ In a ferrofluid one observes similar phenomena but, due to fact that the particles are permanently magnetized, the aggregation takes place even without an applied magnetic field.

${ }^{11}$ S. Miyazima, P. Meakin, and F. Family, Phys. Rev. A 36, 1421 (1987).

${ }^{12}$ G. Helgesen, A.T. Skjeltorp, P.M. Mors, R. Botet, and R. Jullien, Phys. Rev. Lett. 61, 1736 (1988).

13 G. Bossis and J.F. Brady, J. Chem. Phys. 91, 1866 (1989). 
14 S. Fraden, A.J. Hurd, and R.B. Meyer, Phys. Rev. Lett. 63, 2373 (1989).

${ }^{15}$ M. Fermigier and A.P. Gast, J. Colloid and Interface Sci. 154, 522 (1992).

${ }^{16}$ D.J. Klingenberg, C.F. Zukoski, and J.C. Hill, J. Appl. Phys. 73, 4644 (1993).

${ }^{17}$ E. Lemaire, Y. Grasselli and G. Bossis, J. Phys. II France 2, 359 (1992); J. Phys. II France 4, 253 (1994).

18 J.H.E. Promislow, A.P. Gast, and M. Fermigier, J. Chem. Phys. 102, 5492 (1995).

19 M.C. Miguel and R. Pastor-Satorras, submitted to Phys. Rev. E, (1998).

${ }^{20}$ P.G. de Gennes and P. Pincus, Phys. Kondens. Mater. 11, 189 (1970).

${ }^{21}$ H. Wang, Y. Zhu, C. Boyd, W. Luo, A. Cebers, and R.E. Rosensweig, Phys. Rev. Lett. 72, 1929 (1994).

${ }^{22}$ P. Jund, S.G. Kim, D. Tománek and J. Hetherington, Phys. Rev. Lett. 74, 3049 (1995).

${ }^{23}$ In a suspension of superparamagnetic particles, the induced moment is given by $m=\chi H V_{p}$, with $\chi$ the effective magnetic susceptibility of the particle. For the monodomain magnetic particles constituting a ferrofluid $m=M_{s} V_{p}$, with $M_{s}$ the saturation magnetization of the ferromagnetic material, and $V_{p}$ the particle volume. In the latter case, only when $\mu \rightarrow \infty$ all dipoles will be aligned parallel to the external field.

${ }^{24}$ We disregard rotational contributions to the diffusion equation. In the presence of a strong external field, the magnetic moment, rigidly attached to a ferromagnetic particle, is oriented along the field direction, hindering the particle rotation. This is no longer the case in a magnetorheological fluid containing field-induced dipolar particles. Nevertheless, in the ideal situation of an induced moment completely detached from the body of the host particle, and in the absence of hydrodynamic interactions, the translational and rotational dynamics of such spheres are decoupled.

${ }^{25}$ R.B. Bird, O. Hassager, R.C. Armstrong and C.F.Curtis, Dynamics of Polymeric Liquids, (Wiley, New York, 1976).

26 As for polymers, one can introduce a correlation or persistence length $\lambda_{p} \sim \lambda d$. A rigid chain will then be characterized by $\lambda_{p} \gg N d$, or $\lambda \gg N$.

${ }^{27}$ M. Doi and S.F. Edwards, The Theory of Polymer Dynamics, (Oxford University Press, New York, 1989). 